\title{
Differential equations with discrete state-dependent delay: uniqueness and well-posedness in the space of continuous functions
}

\author{
Alexander V. Rezounenko \\ Department of Mechanics and Mathematics, Kharkov University, \\ 4, Svobody Sqr., Kharkov, 61077, Ukraine \\ E-mail: rezounenko@univer.kharkov.ua
}

\begin{abstract}
Partial differential equations with discrete (concentrated) statedependent delays in the space of continuous functions are investigated. In general, the corresponding initial value problem is not well posed, so we find an additional assumption on the state-dependent delay function to guarantee the well posedness. For the constructed dynamical system we study the long-time asymptotic behavior and prove the existence of a compact global attractor.
\end{abstract}

Key words : Partial functional differential equation, state-dependent delay, delay selection, well-possedness, global attractor

Mathematics Subject Classification 2000 : 35R10, 35B41, 35K57.

\section{Introduction}

Feb 4, 2008

Delay differential equations is one of the oldest branches of the theory of infinite dimensional dynamical systems - theory which describes qualitative properties of systems, changing in time.

We refer to the classical monographs on the theory of ordinary (O.D.E.) delay equations Hale (1977), Hale and Lunel (1993), Diekmann et al. (1995), Azbelev et al. (1991), Mishkis (1972)]. The theory of partial (P.D.E.) delay equations is essentially less developed since such equations are infinite-dimensional in both time (as delay equations) and space (as P.D.E.s) variables, which makes the analysis more difficult. We refer to some works which are close to the present research [Travis and Webb (1974), Chueshov (1992), Chueshov and Rezounenko (1995), Boutet de Monvel et al. (1998), Rezounenko (2003) and to the monograph [Wu (1996)].

Recently, a new class of delay equations - equations with a state-dependent delay (SDD) attracts much attention of researchers (see e.g. Walther (2002), Walther (2003), Nussbaum and Mallet-Paret (1992), Nussbaum and Mallet-Paret (1996), Mallet-Paret et al. (1994), Krisztin (2003), Walther (2007)] and also the review [Hartung et al (2006)] for details and references). Investigations of these equations essentially differ from the ones of equations with constant or time-dependent delays. The main difficulty is that nonlinearities with SDD (in contrast to constant or time-dependent delays) are not Lipschitz continuous on the space of continuous functions - the main space of initial data, where the classical theory of delay equations is developed (see the references above). As a consequence, the corresponding initial value problem (IVP), in general, is not well posed (in the sense of J. Hadamard [Hadamard (1902), Hadamard (1932)]). An explicit example 
of the nonuniqueness of solutions to an ordinary equation with state-dependent delay (SDD) is given in Winston (1970)] (see also Hartung et al (2006), p.465]). As noticed in Hartung et al (2006), p.465] "typically, the IVP is uniquely solved for initial and other data which satisfy suitable Lipschitz conditions." The idea to investigate SDD O.D.E.s in the space of Lipschitz continuous functions is very fruitful (see the references above).

Unfortunately, in contrast to O.D.E.s with state-dependent delay, one has no possibility to exploit the space of Lipschitz continuous functions for P.D.E.s with SD delay, because solutions of P.D.E.s usually do not belong to this space.

The first attempt to treat P.D.E.s with state-dependent (state-selective) delays has been made for a distributed delay problem Rezounenko and Wu (2006), Rezounenko (2007)]. The existence (without uniqueness) of solutions for P.D.E.s with discrete state-dependent delay was studied in Hernandez et al (2006)] (mild solutions) and [Rezounenko (2007)] (weak solutions).

As noticed above, it is a common point of view Hartung et al (2006)] that equations (O.D.E.s and P.D.E.s) with discrete state-dependent delay are not well posed in the space of continuous functions (C). This leads to the search of (particular) classes of equations which may be well-posed in the space of continuous functions (C).

The main goal of the present paper is to propose an assumption on the delay, which is sufficient for the well-posedness of the corresponding initial value problem in the space $\mathrm{C}$. To the best of our knowledge, this is the first result for the well-posedness in $\mathrm{C}$ of the discrete state-dependent delay (for P.D.E.s as well as for O.D.E.s).

Discussing the meaning of the main assumption $(\mathrm{H})$ (see below) for applied problems, we hope that the assumption is the natural mathematical expression of the fact that for many applied problems, the models have a parameter (time $\eta_{i g n}>0$ ) which is necessary to take into considerations the time changes in the system. The changes not always can be taken into considerations immediately. To this end, the existence of $\eta_{\text {ign }}>0$ (no matter how small the value of $\eta_{i g n}>0$ is!) makes the corresponding initial value problem well posed.

\section{Formulation of the model with state-dependent discrete delay}

Our goal is to present an approach to study the following partial differential equation with state-dependent discrete delay

$$
\frac{\partial}{\partial t} u(t, x)+A u(t, x)+d u(t, x)=\int_{\Omega} b\left(u\left(t-\eta\left(u_{t}\right), y\right)\right) f(x-y) d y \equiv\left(F\left(u_{t}\right)\right)(x), \quad x \in \Omega,
$$

where $A$ is a densely-defined self-adjoint positive linear operator with domain $D(A) \subset$ $L^{2}(\Omega)$ and with compact resolvent, so $A: D(A) \rightarrow L^{2}(\Omega)$ generates an analytic semigroup, $\Omega$ is a smooth bounded domain in $R^{n_{0}}, f: \Omega-\Omega \rightarrow R$ is a bounded function to be specified later, $b: R \rightarrow R$ is a locally Lipschitz map, $d$ is a non-negative constant. The function $\eta(\cdot): C\left([-r, 0] ; L^{2}(\Omega)\right) \rightarrow R_{+}$represents the state-dependent discrete delay. We denote for short $C \equiv C\left([-r, 0] ; L^{2}(\Omega)\right)$. The norms in $L^{2}(\Omega)$ and $C$ are denoted by $\|\cdot\|$ and $\|\cdot\|_{C}$ respectively. As usually for delay equations, we denote by $u_{t}$ the function of $\theta \in[-r, 0]$ by the formula $u_{t} \equiv u_{t}(\theta) \equiv u(t+\theta)$. 
We consider equation (1) with the following initial condition

$$
\left.u\right|_{[-r, 0]}=\varphi \in C \equiv C\left([-r, 0] ; L^{2}(\Omega)\right) .
$$

The methods used in our work can be applied to another types of nonlinear and delay P.D.E.s (as well as O.D.E.s). We choose a particular form of nonlinear delay terms $F$ for simplicity and to illustrate our approach on the diffusive Nicholson's blowflies equation (see the end of the article for more details). Below you will also find a remark (Rem. 11) on the local in space variable problems.

\section{The existence of mild solutions}

In our study we use the standard

Definition 1. A function $u \in C\left([-r, T] ; L^{2}(\Omega)\right)$ is called a mild solution on $[-r, T]$ of the initial value problem (11), (2) if it satisfies (2) and

$$
u(t)=e^{-A t} \varphi(0)+\int_{0}^{t} e^{-A(t-s)}\left\{F\left(u_{s}\right)-d \cdot u(s)\right\} d s, \quad t \in[0, T] .
$$

Proposition 1. Assume the function $b: R \rightarrow R$ is a locally Lipschitz map, satisfying $|b(w)| \leq C_{1}|w|+C_{b}$ with $C_{i} \geq 0$, and function $\eta(\cdot): C\left([-r, 0] ; L^{2}(\Omega)\right) \rightarrow R_{+}$is continuous, $f: \Omega-\Omega \rightarrow R$ is a bounded function. Then for any initial function $\varphi \in C$, initial value problem (1), (2) has a global mild solution which satisfies $u \in C\left([-r,+\infty) ; L^{2}(\Omega)\right)$.

The existence of a mild solution is a consequence of the continuity of $F(\varphi) \equiv$ $\int_{\Omega} b(\varphi(-\eta(\varphi), y)) f(\cdot-y) d y: C \rightarrow L^{2}(\Omega)$ (see (10) which gives the possibility to use the standard method based on Schauder fixed point theorem (see e.g. Wu (1996), theorem 2.1, p.46]). The solution is also global (is defined for all $t \geq-r$ ) see e.g. $(\mathrm{Wu}(1996)$, theorem 2.3, p.49].

Remark 1. It is important to notice that even in the case of ordinary differential equations (even scalar) the mapping of the form $\widetilde{F}(\varphi)=f(\varphi(-r(\varphi))): C\left(\left[-r_{0}, 0\right] ; R\right) \rightarrow$ $R$ has a very unpleased property. The authors in [Louihi et al (2002), p.3] write "Notice that the functional $\widetilde{F}$ is defined on $C$, but it is clear that it is neither differentiable nor locally Lipschitz continuous, whatever the smoothness of $f$ and $r$." As a consequence, the Cauchy problem associated with equations with such a nonlinearity "...is not well posed in the space of continuous functions, due to the non-uniqueness of solutions whatever the regularity of the functions $f$ and $r$ "Louihi et al (2002), p.2]. See also a detailed discussion in Hartung et al (2006).

\section{Uniqueness and well-posedness}

As in the previous section, we assume that $\eta: C \rightarrow R_{+}$is continuous and $f$ : $\Omega-\Omega \rightarrow R$ is a bounded function $\left(|f(\cdot)| \leq M_{f}\right)$. Additionally, we assume the following assumption on the delay function $\eta$ is satisfied

- $\exists \eta_{\text {ign }}>0$ such that $\eta$ "ignores" values of $\varphi(\theta)$ for $\theta \in\left(-\eta_{i g n}, 0\right]$ i.e.

$$
\exists \eta_{i g n}>0: \forall \varphi^{1}, \varphi^{2} \in C: \forall \theta \in\left[-r,-\eta_{i g n}\right], \Rightarrow \varphi^{1}(\theta)=\varphi^{2}(\theta) \quad \Longrightarrow \quad \eta\left(\varphi^{1}\right)=\eta\left(\varphi^{2}\right) .
$$


Remark 2. It is easy to present many examples of (delay) functions $\eta$, which satisfy assumption (H). Some of them are

$$
\begin{gathered}
\eta(\varphi)=p_{1}(\varphi(-r)) \text { with } p_{1}: L^{2}(\Omega) \rightarrow R_{+} ; \\
\eta(\varphi)=\sum_{k=1}^{N} p_{k}\left(\varphi\left(-r_{k}\right)\right) \text { with } p_{k}: L^{2}(\Omega) \rightarrow R_{+} ; \quad \min r_{k}>0 ; \\
\eta(\varphi)=\int_{-r}^{-\eta_{i g n}} p_{1}(\varphi(\theta)) d \theta, \quad \text { and } \quad \eta(\varphi)=p_{1}\left(\int_{-r}^{-\eta_{i g n}} \varphi(\theta) d \theta\right), \quad \eta_{i g n}>0, \text { etc. }
\end{gathered}
$$

Remark 3. It is interesting to notice that in the case $\eta_{\text {ign }}>r$, we have that the delay function $\eta$ ignores all values of $\varphi(\theta), \forall \theta \in[-r, 0]$, so $\eta(\varphi) \equiv$ const, $\forall \varphi \in C$ i.e. equation (11) becomes equation with constant delay. On the other hand, assumption, similar to (H) with $\eta_{\text {ign }}=0$, is trivial since $\varphi^{1}(\theta)=\varphi^{2}(\theta)$ for all $\theta \in[-r, 0]$ means $\varphi^{1}=\varphi^{2}$ in $C$, so $\eta\left(\varphi^{1}\right)=\eta\left(\varphi^{2}\right)$.

To show that assumption $(\mathrm{H})$ implies the uniqueness of mild solutions (given by Proposition 1), we will use the standard method of steps with a step less than $\eta_{i g n}>0$. First, let us introduce, for any $\varphi \in C$ the extension function

$$
\bar{\varphi}(s) \equiv\left[\begin{array}{ll}
\varphi(s) & s \in[-r, 0] \\
\varphi(0) & s \in\left(0, \eta_{i g n}\right)
\end{array} .\right.
$$

Consider any mild solution $u(t)$ of IVP (11), (2) and the nonlinearity $\int_{\Omega} b(u(t-$ $\left.\left.\eta\left(u_{t}\right), y\right)\right) f(\cdot-y) d y$. For all $t \in\left[0, \eta_{\text {ign }}\right)$ assumption $(\mathrm{H})$ gives $\eta\left(u_{t}\right)=\eta\left(\bar{\varphi}_{t}\right)$. Let us denote by $r^{\varphi}(t) \equiv \eta\left(\bar{\varphi}_{t}\right), t \in\left[0, \eta_{\text {ign }}\right)$.

Hence any mild solution $u(t)$ of IVP (1), (21) for all values of $t \in\left[0, \eta_{\text {ign }}\right)$ is also a solution of

$$
\left\{\begin{array}{l}
\dot{u}(t)+A u(t)+d \cdot u(t)=\int_{\Omega} b\left(u\left(t-r^{\varphi}(t), y\right)\right) f(\cdot-y) d y, \quad t \in\left[0, \eta_{i g n}\right), \\
u(\theta)=\varphi(\theta), \theta \in[-r, 0],
\end{array}\right.
$$

where $r^{\varphi}(t)$ is time-dependent (but not state-dependent delay) known function for all $t \in\left[0, \eta_{i g n}\right)$. To show that the last Cauchy problem (with time-dependent delay) has the unique solution, it is sufficient to consider any two solutions of (44) and their difference $w(t) \equiv u^{1}(t)-u^{2}(t)$, which satisfies (c.f. (3i))

$$
\begin{gathered}
w(t)=\int_{0}^{t} e^{-A(t-s)} \times \\
\left\{\int_{\Omega}\left[b\left(u^{1}\left(s-r^{\varphi}(s), y\right)\right)-b\left(u^{2}\left(s-r^{\varphi}(s), y\right)\right)\right] f(\cdot-y) d y-d \cdot w(s)\right\} d s, t \in\left[0, \eta_{\text {ign }}\right) .
\end{gathered}
$$

An easy calculation, the local Lipschitz property of $b$ and $\left\|e^{-A(t-s)}\right\| \leq 1$ give

$$
\|w(t)\| \leq \int_{0}^{t}\left(M_{f}|\Omega| L_{b}+d\right) \max _{s \in[0, t]}\|w(s)\| d s \leq t \cdot\left(M_{f}|\Omega| L_{b}+d\right) \max _{s \in[0, t]}\|w(s)\| .
$$

Here we denote $|\Omega| \equiv \int_{\Omega} 1 \cdot d x$. 
Remark 4. Here we used that $\max _{s \in[-r, t]}\|w(s)\|=\max _{s \in[0, t]}\|w(s)\|$ since $w(s) \equiv 0$ for $\theta \in[-r, 0]$ ( $w$ is the difference of two solutions, both satisfying (2)).

Choose small enough $\alpha>0$ to satisfy $\alpha\left(M_{f}|\Omega| L_{b}+d\right)<1$. The last estimate gives

$$
\max _{s \in[0, \alpha]}\|w(s)\| \leq \alpha \cdot\left(M_{f}|\Omega| L_{b}+d\right) \max _{s \in[0, \alpha]}\|w(s)\|<\max _{s \in[0, \alpha]}\|w(s)\|
$$

which implies $\max _{s \in[0, \alpha]}\|w(s)\|=0$. This means that any two mild solutions of (4) coincide for $t \in[0, \alpha]$ with $\alpha<\left(M_{f}|\Omega| L_{b}+d\right)^{-1}$. Repeat this considerations (if necessary) by steps of length $\alpha$ to cover $\left[0, \eta_{i g n}\right)$. This gives the uniqueness of solutions of (41) and hence the uniqueness of solutions of (11), (2) for $t \in\left[0, \eta_{i g n}\right)$. The uniqueness on any interval $[0, T]$ holds by the similar arguments on each time interval $\left[k \cdot \eta_{i g n},(k+1)\right.$. $\eta_{\text {ign }}$ ), $k \in N$ (redefining the function $r^{\varphi}(t)$ ).

We may define the evolution operator $S_{t}: C \rightarrow C$ by the formula $S_{t} \varphi \equiv u_{t}$, where $u(t)$ is the unique mild solution of (1), (2) with initial function $\varphi$.

Remark 5. The system becomes much simpler if we additionally assume that the delay function $\eta$ satisfies

$$
\exists \eta_{\min }>0 \text { such that } \eta: C \rightarrow\left[\eta_{\min }, r\right] \text { is continuous. }
$$

In that case we may use the classical method of steps with a step less than $\min \left\{\eta_{\text {ign }}, \eta_{\text {min }}\right\}$. To satisfy assumption (H1) for the functions given in Remark 2 it is sufficient to assume that $\inf p_{i}(\cdot)>0$.

Remark 6. For applied problems described by ordinary differential equations, condition $\eta(\cdot) \in\left[\eta_{\text {min }}, r\right]$ (see (H1)) is used and motivated in [Al-Omari, Gourley (2005), p.15] and [Aiello, Freedmand and Wu (1992)]. The authors write "This assumption is known to be realistic in the case of Antarctic whale and seal populations."

Remark 7. It is interesting to notice that without (H1), one cannot say that the nonlinearity $F\left(u_{t}\right)$ depends on $\left.u\right|_{\left[-r,-\eta_{i g n}\right]}$ only. The SD-delay may vanish (i.e. (H1) does not hold).

The main result of this section is the following

Theorem 1. Assume the function $b: R \rightarrow R$ is a locally Lipschitz map, satisfying $|b(w)| \leq C_{1}|w|+C_{b}$ with $C_{i} \geq 0$, the delay function $\eta: C \rightarrow R_{+}$is continuous and satisfies the assumption (H), $f: \Omega-\Omega \rightarrow R$ is a bounded function $\left(|f(z)| \leq M_{f}, \forall z \in\right.$ $\Omega-\Omega)$. Then the pair $\left(S_{t}, C\right)$ constitutes a dynamical system i.e. the following properties are satisfied:

1. $S_{0}=I d($ identity operator in $C)$;

2. $\forall t, \tau \geq 0 \quad \Longrightarrow \quad S_{t} S_{\tau}=S_{t+\tau}$;

3. $t \mapsto S_{t}$ is a strongly continuous in $C$ mapping;

4. for any $t \geq 0$ the evolution operator $S_{t}$ is continuous in $C$ i.e. for any $\left\{\varphi^{n}\right\}_{n=1}^{\infty} \subset C$ such that $\left\|\varphi^{n}-\varphi\right\|_{C} \rightarrow 0$ as $n \rightarrow \infty$, one has $\left\|S_{t} \varphi^{n}-S_{t} \varphi\right\|_{C} \rightarrow 0$ as $n \rightarrow \infty$. 
Remark 8. Theorem 1 particularly means that the initial value problem (1), (2) is well posed in the space $C$ in the sense of J. Hadamard [Hadamard (1902), Hadamard (1932)].

Remark 9. It is important to emphasize, that we do not assume the SD-delay function $\eta$ to be Lipschitz (c.f. [Rezounenko (2007)]). We propose an alternative approach, based on the assumption (H) which is of different nature to the Lipschitz property of $\eta$.

Proof. Properties 1), 2) are consequences of the uniqueness of mild solutions due to $(\mathrm{H})$ (see considerations above). Property 3) is given by Proposition 1 since the solution is a continuous function $u \in C\left([-r, T] ; L^{2}(\Omega)\right)$.

Let us prove property 4. Let us fix $t_{0} \in\left[0, \eta_{i g n}\right)$. Denote by $u^{k}(t)$ the solution of (11),(2) with the initial function $\varphi^{k}$ and by $u(t)$ the solution of (11),(2) with the initial function $\varphi$.

We use the variation of constants formula for parabolic equation (4) (with $\widetilde{A} \equiv A+d \cdot$ )

$$
\begin{gathered}
u(t)=e^{-\widetilde{A} t} u(0)+\int_{0}^{t} e^{-\widetilde{A}(t-\tau)} \int_{\Omega} b\left(u\left(\tau-\eta\left(u_{\tau}\right), y\right)\right) f(\cdot-y) d y d \tau \\
u^{k}(t)=e^{-\widetilde{A} t} u^{k}\left((0)+\int_{0}^{t} e^{-\widetilde{A}(t-\tau)} \int_{\Omega} b\left(u^{k}\left(\tau-\eta\left(u_{\tau}^{k}\right), y\right)\right) f(\cdot-y) d y d \tau .\right.
\end{gathered}
$$

Using $\left\|e^{-\widetilde{A} t}\right\| \leq 1$ and $\left\|e^{-\widetilde{A}(t-\tau)}\right\| \leq 1$, we get

$$
\begin{gathered}
\left\|u^{k}(t)-u(t)\right\| \leq\left\|u^{k}(0)-u(0)\right\| \\
+\int_{0}^{t}\left\|\int_{\Omega}\left[b\left(u^{k}\left(\tau-\eta\left(u_{\tau}^{k}\right), y\right)\right)-b\left(u\left(\tau-\eta\left(u_{\tau}\right), y\right)\right)\right] f(\cdot-y) d y\right\| d \tau \\
=\left\|\varphi^{k}(0)-\varphi(0)\right\|+\mathcal{J}_{1}^{k}(t)+\mathcal{J}_{2}^{k}(t)
\end{gathered}
$$

where we denote

$$
\begin{gathered}
\mathcal{J}_{1}^{k}(s) \equiv \int_{0}^{s}\left\|\int_{\Omega}\left[b\left(u^{k}\left(\tau-\eta\left(u_{\tau}^{k}\right), y\right)\right)-b\left(u\left(\tau-\eta\left(u_{\tau}^{k}\right), y\right)\right)\right] f(\cdot-y) d y\right\| d \tau, \\
\mathcal{J}_{2}^{k}(s) \equiv \int_{0}^{s}\left\|\int_{\Omega}\left[b\left(u\left(\tau-\eta\left(u_{\tau}^{k}\right), y\right)\right)-b\left(u\left(\tau-\eta\left(u_{\tau}\right), y\right)\right)\right] f(\cdot-y) d y\right\| d \tau .
\end{gathered}
$$

Using the Lipschitz property of $b$, one easily gets

$\mathcal{J}_{1}^{k}(t) \leq M_{f}|\Omega| L_{b} \int_{0}^{t}\left\|u^{k}\left(\tau-\eta\left(u_{\tau}^{k}\right)\right)-u\left(\tau-\eta\left(u_{\tau}^{k}\right)\right)\right\| d \tau \leq M_{f}|\Omega| L_{b} t \max _{s \in[-r, t]}|| u^{k}(s)-u(s) \|$.

Estimates (11), (18) and property $\mathcal{J}_{2}^{k}(s) \leq \mathcal{J}_{2}^{k}(t)$ for $s \leq t \leq t_{0}<\eta_{\text {ign }}$ give

$$
\max _{t \in\left[0, t_{0}\right]}\left\|u^{k}(t)-u(t)\right\| \leq\left\|\varphi^{k}(0)-\varphi(0)\right\|+M_{f}|\Omega| L_{b} t_{0} \max _{s \in\left[-r, t_{0}\right]}\left\|u^{k}(s)-u(s)\right\|+\mathcal{J}_{2}^{k}\left(t_{0}\right) .
$$

Hence

$$
\max _{s \in\left[-r, t_{0}\right]}\left\|u^{k}(s)-u(s)\right\| \leq\left\|\varphi^{k}-\varphi\right\|_{C}+M_{f}|\Omega| L_{b} t_{0} \max _{s \in\left[-r, t_{0}\right]}\left\|u^{k}(s)-u(s)\right\|+\mathcal{J}_{2}^{k}\left(t_{0}\right) .
$$


We choose $t_{0}<\left[M_{f}|\Omega| L_{b}\right]^{-1}$ (to satisfy $M_{f}|\Omega| L_{b} t_{0}<1$ ) and get

$$
\left(1-M_{f}|\Omega| L_{b} t_{0}\right) \max _{s \in\left[-r, t_{0}\right]}\left\|u^{k}(s)-u(s)\right\| \leq\left\|\varphi^{k}-\varphi\right\|_{C}+\mathcal{J}_{2}^{k}\left(t_{0}\right)
$$

Our goal is to show that $\mathcal{J}_{2}^{k}\left(t_{0}\right) \rightarrow 0$ as $k \rightarrow \infty$. The Lipschitz property of $b$ implies

$$
\mathcal{J}_{2}^{k}\left(t_{0}\right) \leq M_{f}|\Omega| L_{b} \int_{0}^{t_{0}}\left\|u\left(\tau-\eta\left(u_{\tau}^{k}\right)\right)-u\left(\tau-\eta\left(u_{\tau}\right)\right)\right\| d \tau
$$

We use the extension functions

$$
\bar{\varphi}(s) \equiv\left[\begin{array} { l l } 
{ \varphi ( s ) } & { s \in [ - r , 0 ] ; } \\
{ \varphi ( 0 ) } & { s \in ( 0 , \eta _ { \text { ign } } ) }
\end{array} \quad \text { and } \quad \overline { \varphi } ^ { k } ( s ) \equiv \left[\begin{array}{cc}
\varphi^{k}(s) & s \in[-r, 0] \\
\varphi^{k}(0) & s \in\left(0, \eta_{\text {ign }}\right)
\end{array}\right.\right.
$$

It is easy to see that the convergence $\left\|\varphi^{k}-\varphi\right\|_{C} \rightarrow 0$ implies $\left\|\bar{\varphi}_{\tau}^{k}-\bar{\varphi}_{\tau}\right\|_{C} \rightarrow 0$ for any $\tau \in\left[0, \eta_{\text {ign }}\right)$.

On the other hand, for any $\tau \in\left[0, \eta_{\text {ign }}\right]$ and any $\theta \in\left[-r,-\eta_{\text {ign }}\right]$ we have $u_{\tau}^{k}(\theta)=$ $\varphi^{k}(\tau+\theta)$, hence assumption (H) gives $\eta\left(u_{\tau}^{k}\right)=\eta\left(\bar{\varphi}_{\tau}^{k}\right)$ for any $\tau \in\left[0, \eta_{i g n}\right)$. The same arguments give $\eta\left(u_{\tau}\right)=\eta\left(\bar{\varphi}_{\tau}\right)$ for any $\tau \in\left[0, \eta_{i g n}\right)$.

The considerations above show that the convergence $\left\|\varphi^{k}-\varphi\right\|_{C} \rightarrow 0$ implies $\mid \eta\left(u_{\tau}^{k}\right)-$ $\eta\left(u_{\tau}\right)|=| \eta\left(\bar{\varphi}_{\tau}^{k}\right)-\eta\left(\bar{\varphi}_{\tau}\right) \mid \rightarrow 0$ for all $\tau \in\left[0, \eta_{i g n}\right)$. Here we used the continuity of $\eta: C \rightarrow R_{+}$.

The last property gives that for all $\tau \in\left[0, \eta_{i g n}\right)$ one has $\tau-\eta\left(u_{\tau}^{k}\right) \rightarrow \tau-\eta\left(u_{\tau}\right)$, when $k \rightarrow \infty$. Hence the continuity of the mild solution $u$ (the strong continuity in $L^{2}(\Omega)$ ) implies (see the integral in (13))

$$
\forall \tau \in\left[0, t_{0}\right] \Longrightarrow\left\|u\left(\tau-\eta\left(u_{\tau}^{k}\right)\right)-u\left(\tau-\eta\left(u_{\tau}\right)\right)\right\| \rightarrow 0, \quad \text { when } \quad k \rightarrow \infty .
$$

On the other hand, it is evidently that

$$
\forall \tau \in\left[0, t_{0}\right] \Longrightarrow\left\|u\left(\tau-\eta\left(u_{\tau}^{k}\right)\right)-u\left(\tau-\eta\left(u_{\tau}\right)\right)\right\| \leq 2 \max _{s \in\left[-r, t_{0}\right]}\|u(s)\|<+\infty .
$$

Properties (14) and (15) allow us to use Lebesgue-Fatou lemma ([Yosida (1965), p.32]) for the scalar function $\left\|u\left(\tau-\eta\left(u_{\tau}^{k}\right)\right)-u\left(\tau-\eta\left(u_{\tau}\right)\right)\right\|$ to conclude that

$$
\int_{0}^{t_{0}}\left\|u\left(\tau-\eta\left(u_{\tau}^{k}\right)\right)-u\left(\tau-\eta\left(u_{\tau}\right)\right)\right\| d \tau \rightarrow 0 \quad \text { when } \quad k \rightarrow \infty .
$$

Estimates (16) and (13) prove that $\mathcal{J}_{2}^{k}\left(t_{0}\right) \rightarrow 0$ as $k \rightarrow \infty$.

Since

$$
\max _{t \in\left[0, t_{0}\right]} \mathcal{J}_{2}^{k}(t) \leq \mathcal{J}_{2}^{k}\left(t_{0}\right) \rightarrow 0 \quad \text { as } k \rightarrow \infty,
$$

we finally conclude (see (12) and the last properties) that for all $t \in\left[0, t_{0}\right]$ :

$$
\begin{gathered}
\left\|u_{t}^{k}-u_{t}\right\|_{C} \equiv \max _{\theta \in[-r, 0]}\left\|u^{k}(t+\theta)-u(t+\theta)\right\|_{L^{2}(\Omega)} \\
\leq\left[1-M_{f}|\Omega| L_{b} t_{0}\right]^{-1} \cdot\left(\left\|\varphi^{k}-\varphi\right\|_{C}+\mathcal{J}_{2}^{k}\left(t_{0}\right)\right) \rightarrow 0 \quad \text { as } k \rightarrow \infty .
\end{gathered}
$$


Estimate (17) gives that $\left\|u_{t}^{k}-u_{t}\right\|_{C} \rightarrow 0$ when $\left\|\varphi^{k}-\varphi\right\|_{C} \rightarrow 0$ for all $t \in\left[0, t_{0}\right] \subset$ $\left[0, \min \left\{\left[M_{f}|\Omega| L_{b}\right]^{-1}, \eta_{i g n}\right\}\right)$. This is the strong continuity in the space $C$ of the evolution operator $S_{t}$ for all (small) $t \in\left[0, t_{0}\right] \subset\left[0, \min \left\{\left[M_{f}|\Omega| L_{b}\right]^{-1}, \eta_{\text {ign }}\right\}\right.$ ).

For any $t \geq 0$ we present $S_{t} \varphi$ as the composition of mappings

$S_{t} \varphi=\underbrace{S_{p} \circ S_{p} \circ S_{p} \circ \ldots \circ S_{p}}_{q \text { times }} \circ S_{t-\left[t \cdot(2 p)^{-1}\right]} \varphi, \quad$ where $\quad p \equiv \frac{1}{2} \min \left\{L_{b}^{-1}, \eta_{\text {ign }}\right\}, \quad q \equiv\left[\frac{t}{2 p}\right]$.

Here [.] denotes the integer part of a real number. The continuity of $S_{t}$ follows from the proved continuity of $S_{p}$ and $S_{t-\left[t \cdot(2 p)^{-1}\right]}$ (since both $p$ and $t-\left[t \cdot(2 p)^{-1}\right]$ belong to $\left.\left[0, \min \left\{\left[M_{f}|\Omega| L_{b}\right]^{-1}, \eta_{i g n}\right\}\right)\right)$. The property 4 is proved. It complies the proof of Theorem 1.

\section{Asymptotic behavior}

This section is devoted to the study of the long-time asymptotic behavior of the dynamical system $\left(S_{t}, C\right)$, constructed in Theorem 1 .

Theorem 2. Assume the function $b: R \rightarrow R$ is a locally Lipschitz bounded map $\left(|b(w)| \leq C_{b}\right.$ with $\left.C_{b} \geq 0\right)$ and the delay function $\eta: C \rightarrow R_{+}$is continuous and satisfies the assumption (H), $f: \Omega-\Omega \rightarrow R$ is a bounded function $\left(|f(\cdot)| \leq M_{f}\right)$. Then the dynamical system $\left(S_{t}, C\right)$ has a compact global attractor which is a compact set in all spaces $C_{\delta} \equiv C\left([-r, 0] ; D\left(A^{\delta}\right)\right), \forall \delta \in\left[0, \frac{1}{2}\right)$.

Proof. Our proof is split on four steps.

Step 1. Let us first prove that for any $T>0$ and any bounded set $B \subset C$ there exists a constant $C_{T}(B)$ such that for any mild solution of (1), (2) with initial values in $B$, one has

$$
\forall T>0, \forall B, \exists C_{T}(B)>0: \forall t \in[0, T] \Longrightarrow\|u(t)\| \leq C_{T}(B) .
$$

Equation (3) implies $\|u(t)\| \leq\|\varphi(0)\|+\int_{0}^{t}\left(\left\|F\left(u_{s}\right)\right\|+d\|u(s)\|\right) d s$. Using $\left\|F\left(u_{s}\right)\right\| \leq$ $M_{f}|\Omega|^{3 / 2} C_{b}$, we have $\|u(t)\| \leq\|\varphi(0)\|+d \int_{0}^{t}\|u(s)\| d s+t M_{f}|\Omega|^{3 / 2} C_{b}$. Denote by $\Psi(t) \equiv$ $\int_{0}^{t}\|u(s)\| d s$ and use Gronwall lemma to get $\Psi(t) \leq \| \varphi(0)|| d^{-1} e^{d t}+e^{d t} M_{f}|\Omega|^{3 / 2} C_{b} d^{-2}[1-$ $\left.e^{-d t}(d t-1)\right]$. The last estimate gives

$\|u(t)\| \leq\|\varphi(0)\|+d \cdot\left[\|\varphi(0)\| d^{-1} e^{d t}+e^{d t} M_{f}|\Omega|^{3 / 2} C_{b} d^{-2}\left[1-e^{-d t}(d t-1)\right]\right]+t M_{f}|\Omega|^{3 / 2} C_{b}$.

It implies (19).

Step 2. Next we show that a solution becomes more smooth for positive $t$.

Lemma. For any $0<\alpha<1, \epsilon>0, T>0$ and any bounded set $B \subset C$ there exists a constant $C_{\alpha, \epsilon, T}(B)$ such that for any mild solution of (1), (2) with initial values in $B$, one has

$$
\left\|A^{\alpha} u(t)\right\| \leq C_{\alpha, \epsilon, T}(B) \quad \text { for } t \in(\epsilon, T] .
$$

The proof of the lemma is standard (see e.g. Chueshov (1999)] and also RezounenkoMAG-1997, Rezounenko-Wu-2006). 
Step 3. Dissipativness. Lemma implies that $u(t) \in D\left(A^{\frac{1}{2}+\delta}\right), \forall \delta \in[0,1 / 2), t \geq$ $\varepsilon(\delta)$. Hence $A u(t) \in D\left(A^{-\frac{1}{2}+\delta}\right), \forall \delta \in[0,1 / 2), t \geq \varepsilon(\delta)$. Equation (11) gives $\dot{u}(t) \in$ $D\left(A^{-\frac{1}{2}+\delta}\right), \forall \delta \in[0,1 / 2), t \geq \varepsilon(\delta)$. So for all $t \geq \varepsilon(\delta)$ one has

$$
\begin{aligned}
& \frac{1}{2} \frac{d}{d t}\left\|A^{\delta} u(t)\right\|^{2}+\left\|A^{\frac{1}{2}+\delta} u(t)\right\|^{2}+d \cdot\left\|A^{\delta} u(t)\right\|^{2} \leq\left\|F\left(u_{t}\right)\right\| \cdot\left\|A^{2 \delta} u(t)\right\| \\
& \leq M_{f}|\Omega|^{3 / 2} C_{b} \cdot \lambda_{1}^{2 \delta-1}\left\|A^{\frac{1}{2}+\delta} u(t)\right\| \leq \frac{1}{2} M_{f}^{2}|\Omega|^{3} C_{b}^{2} \lambda_{1}^{4 \delta-2}+\frac{1}{2}\left\|A^{\frac{1}{2}+\delta} u(t)\right\|^{2} .
\end{aligned}
$$

Hence $\frac{d}{d t}\left\|A^{\delta} u(t)\right\|^{2}+\left\|A^{\frac{1}{2}+\delta} u(t)\right\|^{2}+2 d \cdot\left\|A^{\delta} u(t)\right\|^{2} \leq M_{f}^{2}|\Omega|^{3} C_{b}^{2} \lambda_{1}^{4 \delta-2}$. Using $\left\|A^{\frac{1}{2}+\delta} v\right\|^{2} \geq$ $\lambda_{1}\left\|A^{\delta} v\right\|^{2}$ we have $\frac{d}{d t}\left\|A^{\delta} u(t)\right\|^{2}+\left(\lambda_{1}+2 d\right)\left\|A^{\delta} u(t)\right\|^{2} \leq M_{f}^{2}|\Omega|^{3} C_{b}^{2} \lambda_{1}^{4 \delta-2}$. Gronwall's lemma gives $\left\|A^{\delta} u(t)\right\|^{2} \leq\left\|A^{\delta} u(\varepsilon)\right\|^{2} \cdot \exp \left\{-\left(\lambda_{1}+2 d\right) t\right\}+M_{f}^{2}|\Omega|^{3} C_{b}^{2} \lambda_{1}^{4 \delta-2}\left(\lambda_{1}+2 d\right)^{-1}$. By lemma (step 2), the value $\left\|A^{\delta} u(\varepsilon)\right\|$ is finite, which implies that

$$
\begin{gathered}
\forall \delta \in[0,1 / 2) \quad \exists C(\delta)>0: \forall B-\text { bounded in } C, \exists t(B, \delta): \\
\forall t \geq t(B, \delta) \Longrightarrow\left\|A^{\delta} u(t)\right\| \leq C(\delta) .
\end{gathered}
$$

Step 4. Our next step is to show that the set $\left\{S_{t} \varphi \mid \varphi \in B, t>r+\varepsilon\right\}$ is an equicontinuous family in $C_{\delta} \equiv C\left([-r, 0] ; D\left(A^{\delta}\right)\right), \forall \delta \in\left[0, \frac{1}{2}\right)$.

Remark 10. In our case we cannot use [Wu (1996), thm. 1.8, p.42] since our nonlinearity $F$ is not Lipschitz.

We denote by $\left\{e_{k}\right\}_{k=1}^{\infty}$ the orthonormal basis (of $L^{2}(\Omega)$ ) of eigenvectors of the operator $A$, so $A e_{k}=\lambda_{k} e_{k}, 0<\lambda_{1}<\lambda_{2}<\ldots<\lambda_{k} \rightarrow+\infty$. Consider for $v \in L^{2}(\Omega)$

$$
\left\|A^{\delta}\left(e^{-A t_{1}} v-e^{-A t_{2}} v\right)\right\|^{2}=\sum_{k=1}^{\infty}\left(e^{-\lambda_{k} t_{1}}-e^{-\lambda_{k} t_{2}}\right)^{2} \lambda_{k}^{2 \delta} \cdot v_{k}^{2}, \quad \text { where } \quad v_{k} \equiv\left\langle v, e_{k}\right\rangle .
$$

Assuming $0<t_{1}<t_{2}$, one can easily check that $\left(e^{-\mu t_{1}}-e^{-\mu t_{2}}\right)^{2} \mu^{2 \delta}=\left|e^{-\mu t_{1}}-e^{-\mu t_{2}}\right| \cdot$ $\left|\left(e^{-\mu t_{1}}-e^{-\mu t_{2}}\right) \mu^{2 \delta}\right| \leq\left|\left(e^{-\mu t_{1}}-e^{-\mu t_{2}}\right) \mu^{2 \delta}\right| \leq\left|t_{1}-t_{2}\right| \cdot \max _{\tau \in\left[t_{1}, t_{2}\right]} \mu^{1+2 \delta} e^{-\mu \tau}=\left|t_{1}-t_{2}\right| \cdot$ $\mu^{1+2 \delta} e^{-\mu t_{1}}, \mu>0$. Calculations give $\max _{\mu>0} \mu^{1+2 \delta} e^{-\mu t_{1}}=e^{-(1+2 \delta)}\left(\frac{1+2 \delta}{t_{1}}\right)^{1+2 \delta}$. Hence, for any $k \in N$, one has

$$
\left(e^{-\lambda_{k} t_{1}}-e^{-\lambda_{k} t_{2}}\right)^{2} \lambda_{k}^{2 \delta} \leq\left|t_{1}-t_{2}\right| \cdot e^{-(1+2 \delta)}(1+2 \delta)^{(1+2 \delta)} \cdot\left(\min \left\{t_{1}, t_{2}\right\}\right)^{-(1+2 \delta)}
$$

The last estimate and (22) give

$$
\left\|A^{\delta}\left(e^{-A t_{1}} v-e^{-A t_{2}} v\right)\right\| \leq D_{\delta}\left(\min \left\{t_{1}, t_{2}\right\}\right)^{-\left(\delta+\frac{1}{2}\right)} \cdot \sqrt{\left|t_{1}-t_{2}\right|} \cdot\|v\|,
$$

where $D_{\delta} \equiv e^{-\left(\delta+\frac{1}{2}\right)}\left(\delta+\frac{1}{2}\right)^{\left(\delta+\frac{1}{2}\right)}$. In the same way we get

$$
\left\|A^{\delta}\left(e^{-A t_{1}} v-e^{-A t_{2}} v\right)\right\| \leq \widehat{D}_{\delta}\left(\min \left\{t_{1}, t_{2}\right\}\right)^{-(1+\delta)} \cdot\left|t_{1}-t_{2}\right| \cdot\|v\|,
$$

where $\widehat{D}_{\delta} \equiv e^{-(1+\delta)}(1+\delta)^{1+\delta}$ and also (see e.g. [Chueshov (1999), (2.8.6)]), we get $\left\|A^{\delta} e^{-A s}\right\| \leq(e \cdot s)^{-\delta} \delta^{\delta}$. Calculations and the last estimate give for $0<t_{1}<t_{2}$

$$
\int_{t_{1}}^{t_{2}}\left\|A^{\delta} e^{-A\left(t_{2}-\tau\right)}\right\| d \tau \leq\left(\frac{\delta}{e}\right)^{\delta} \cdot \frac{\left|t_{1}-t_{2}\right|^{1-\delta}}{1-\delta}
$$


Let us consider for any mild solution $u(t)$, the function $G(t) \equiv F\left(u_{t}\right)+d u(t)$ and the difference (see (33))

$$
\begin{gathered}
\left\|A^{\delta}\left(u\left(t_{1}\right)-u\left(t_{2}\right)\right)\right\| \leq\left\|A^{\delta}\left(e^{-A t_{1}} \varphi(0)-e^{-A t_{2}} \varphi(0)\right)\right\| \\
+\int_{0}^{t_{1}}\left\|A^{\delta}\left(e^{-A\left(t_{1}-\tau\right)} G(\tau)-e^{-A\left(t_{2}-\tau\right)} G(\tau)\right)\right\| d \tau+\int_{t_{1}}^{t_{2}}\left\|A^{\delta} e^{-A\left(t_{2}-\tau\right)} G(\tau)\right\| d \tau .
\end{gathered}
$$

Here, as before $0<t_{1}<t_{2}$. The last estimate, (23) and (25) give

$$
\left\|A^{\delta}\left(u\left(t_{1}\right)-u\left(t_{2}\right)\right)\right\| \leq L\left(\delta, t_{1}, t_{2}, \varphi\right) \cdot \sqrt{\left|t_{1}-t_{2}\right|},
$$

where

$$
\begin{gathered}
L\left(\delta, t_{1}, t_{2}, \varphi\right) \equiv D_{\delta}\left(\min \left\{t_{1}, t_{2}\right\}\right)^{-\left(\delta+\frac{1}{2}\right)}\|\varphi(0)\| \\
+\left[D_{\delta} t_{1}^{\frac{1}{2}-\delta}\left(\frac{1}{2}-\delta\right)^{-1}+\delta^{\delta}(e(1-\delta))^{-1}\right] \cdot \max _{\tau \in\left[0, t_{2}\right]}\left\|F\left(u_{\tau}\right)+d u(\tau)\right\| .
\end{gathered}
$$

Here we used

$$
\begin{aligned}
& \int_{0}^{t_{1}}\left\|A^{\delta}\left(e^{-A\left(t_{1}-\tau\right)} G(\tau)-e^{-A\left(t_{2}-\tau\right)} G(\tau)\right)\right\| d \tau \\
\leq & D_{\delta} \sqrt{\left|t_{1}-t_{2}\right|} \max _{\tau \in\left[0, t_{2}\right]}\|G(\tau)\| \cdot \int_{0}^{t_{1}}\left(t_{1}-\tau\right)^{-\left(\delta+\frac{1}{2}\right)} d \tau \\
= & D_{\delta} \sqrt{\left|t_{1}-t_{2}\right|} \max _{\tau \in\left[0, t_{2}\right]}\|G(\tau)\| \cdot t_{1}^{\frac{1}{2}-\delta}\left(\frac{1}{2}-\delta\right)^{-1} .
\end{aligned}
$$

It is evidently that (21) gives $\max _{\tau \in\left[0, t_{2}\right]}\left\|F\left(u_{\tau}\right)+d u(\tau)\right\|<C$ for any mild solution which is already in the ball of dissipation i.e. (21) holds. These, the form of the constant $L\left(\delta, t_{1}, t_{2}, \varphi\right)$ (see (27)) and (26) imply that for any time interval $[a, b] \subset(0,+\infty)$ with $a>t(B, \delta)($ see (21) $)$, there exists a constant $L>0$ such that for any mild solution $u(t)$ and any $t_{1}, t_{2} \in[a, b]$ one has

$$
\left\|A^{\delta}\left(u\left(t_{1}\right)-u\left(t_{2}\right)\right)\right\| \leq L \cdot \sqrt{\left|t_{1}-t_{2}\right|} .
$$

This gives the equicontinuity of the family $\{u(t+\theta) \mid \theta \in[-r, 0], t>t(B, \delta)\}$ in all spaces $C_{\delta} \equiv C\left([-r, 0] ; D\left(A^{\delta}\right)\right), \forall \delta \in\left[0, \frac{1}{2}\right)$.

Finally, estimate (28) for $\delta \in\left[0, \frac{1}{2}\right)$ and estimate (21) for $\delta_{1} \in\left(\delta, \frac{1}{2}\right)$, particularly mean (by Arzela-Ascoli theorem) that for any $\varphi \in C$ and $t>t(B, \delta)$ (see (21), one has $S_{t} \varphi \in K_{\delta}$, where $K_{\delta}$ is a compact set in all spaces $C_{\delta} \equiv C\left([-r, 0] ; D\left(A^{\delta}\right)\right), \forall \delta \in\left[0, \frac{1}{2}\right)$.

That means that the dynamical system $\left(S_{t} ; C\right)$ is dissipative and asymptotically compact, hence by the classical theorem on the existence of an attractor (see, for example, [Babin and Vishik (1992), Temam (1988)] $)\left(S_{t} ; C\right)$ has a compact global attractor. The proof of Theorem 2 is complete. 
Remark 11. All the results above are valid for local in space variable nonlinearity i.e. equation (11) with

$$
\left(F_{\ell}\left(u_{t}\right)\right)(x) \equiv b\left(u\left(t-\eta\left(u_{t}\right), x\right)\right), \quad x \in \Omega .
$$

As an application we can consider the diffusive Nicholson's blowflies equation (see e.g. [So and Yang (1998), So, Wu and Yang (2000)] ) with state-dependent delays. More precisely, we consider equation (11) where $-A$ is the Laplace operator with the Dirichlet boundary conditions, $\Omega \subset R^{n_{0}}$ is a bounded domain with a smooth boundary, the function $f$ can be, for example, $f(s)=\frac{1}{\sqrt{4 \pi \alpha}} e^{-s^{2} / 4 \alpha}$, as in So, Wu and Zou (2001)] (for the non-local in space problem) or Dirac delta-function to get the local problem (see Remark 11), the nonlinear function $b$ is given by $b(w)=p \cdot w e^{-w}$. Function $b$ is bounded, so for any continuous delay function $\eta$, satisfying $(\mathrm{H})$, the conditions of Theorems 1,2 are valid. As a result, we conclude that the initial value problem (11),(2) is well posed in $C$ and the dynamical system $\left(S_{t}, C\right)$ has a global attractor (Theorem 2).

Remark 12. All the considerations above are obviously valid for O.D.E.s, for example, of the following form

$$
\dot{u}(t)+A u(t)+d \cdot u(t)=b\left(u\left(t-\eta\left(u_{t}\right)\right)\right), \quad u(\cdot) \in R^{n}, d \geq 0 .
$$

One simply needs to substitute $L^{2}(\Omega)$ by $R^{n}$ and use $C \equiv C\left([-r, 0] ; R^{n}\right)$ instead of $C\left([-r, 0] ; L^{2}(\Omega)\right)$. Assumptions on the delay function $\eta$ are the same. The function $b: R^{n} \rightarrow R^{n}$ is locally Lipschitz continuous and satisfies $\|b(w)\|_{R^{n}} \leq C_{1}\|w\|_{R^{n}}+C_{b}$ with $C_{1}, C_{b} \geq 0$. For the method of steps in the case of O.D.E.s see e.g. WWalther (2002), Proposition 1].

Acknowledgements. The author wishes to thank Professors I.D. Chueshov and H.-O. Walther for useful discussions of an early version of the manuscript.

\section{References}

[Al-Omari, Gourley (2005)] J.F.M. Al-Omary, S.A.Gourley, Dynamics of a statestructured population model incorporating a state-dependent maturation delay, Nonlinear Analysis: Real World Applications, 6 (2005), 13-33.

[Aiello, Freedmand and Wu (1992)] W. G. Aiello, H. I. Freedmand and J. H. Wu, Analysis of a model representing state-structured population growth with statedependent time delay, SIAM J. Appl. Math., 52 (1992), 855-869.

[Azbelev et al. (1991)] N.V. Azbelev, V.P. Maksimov and L.F. Rakhmatullina, Introduction to the theory of functional differential equations, Moscow, Nauka, 1991.

[Babin and Vishik (1992)] A. V. Babin, and M. I. Vishik, Attractors of Evolutionary Equations, Amsterdam, North-Holland, 1992. 
[Boutet de Monvel et al. (1998)] L. Boutet de Monvel, I. D. Chueshov and A. V. Rezounenko, Inertial manifolds for retarded semilinear parabolic equations, Nonlinear Analysis, 34 (1998), 907-925.

[Chueshov (1992)] I. D. Chueshov, On a certain system of equations with delay, occuring in aeroelasticity, J. Soviet Math. 58, 1992, p.385-390.

[Chueshov and Rezounenko (1995)] I. D. Chueshov, A. V. Rezounenko, Global attractors for a class of retarded quasilinear partial differential equations, C.R.Acad.Sci.Paris, Ser.I 321 (1995), 607-612, ( detailed version: Math.Physics, Analysis, Geometry, Vol.2, N.3 (1995), 363-383).

[Chueshov (1999)] I. D. Chueshov, Introduction to the Theory of Infinite-Dimensional Dissipative Systems, Acta, Kharkov (1999), (in Russian). English transl. Acta, Kharkov (2002) (see http://www.emis.de/monographs/Chueshov ).

[Diekmann et al. (1995)] O. Diekmann, S. van Gils, S. Verduyn Lunel, H-O. Walther, Delay Equations: Functional, Complex, and Nonlinear Analysis, Springer-Verlag, New York, 1995.

[Hadamard (1902)] J. Hadamard, Sur les problèmes aux derivees partielles et leur signification physique, Bull. Univ. Princeton (1902), 13.

[Hadamard (1932)] J. Hadamard, Le problème de Cauchy et les èquations aux derivees partielles linéaires hyperboliques, Hermann, Paris, 1932.

[Hale (1977)] J. K. Hale, Theory of Functional Differential Equations, Springer, BerlinHeidelberg- New York, 1977.

[Hale and Lunel (1993)] J. K. Hale and S. M. Verduyn Lunel, Theory of Functional Differential Equations, Springer-Verlag, New York, 1993.

[Hartung et al (2006)] F. Hartung, T. Krisztin, H.-O. Walther, J. Wu, Functional Differential Equations with State-Dependent Delays: Theory and Applications, in "Handbook of Differential Equations: Ordinary Differential Equations, Volume 3" (A. Canada, P. Drabek, A. Fonda eds.), Elsevier B.V., 2006.

[Hernandez et al (2006)] E.Hernandez, A.Prokopczyk, L.Ladeira, A note on partial functional differential equations with state-dependent delay, Nonlinear Anal. R.W.A. 7, -2006, no. 4, 510-519.

[Krisztin et al. (1999)] T. Krisztin, H.-O. Walther and J. Wu, Shape, Smoothness and Invariant Stratification of an Attracting Set for Delayed Monotone Positive Feedback, Fields Institute Monographs, 11, AMS, Providence, RI, 1999.

[Krisztin (2003)] T. Krisztin, A local unstable manifold for differential equations with state-dependent delay, Discrete Cintin. Dyn. Syst. 9, 933-1028. 
[Louihi et al (2002)] M. Louihi, M.L. Hbid, O. Arino, Semigroup properties and the Crandall Liggett approximation for a class of differential equations with statedependent delays, Journal of Differential Equations 181 (2002), 1-30.

[Mishkis (1972)] A.D. Mishkis, Linear differential equations with retarded argument. 2nd edition, Nauka, Moscow, 1972.

[Nussbaum and Mallet-Paret (1992)] J. Mallet-Paret and R. D. Nussbaum, Boundary layer phenomena for differential-delay equations with state-dependent time lags I, Archive for Rational Mechanics and Analysis 120 (1992), 99-146.

[Nussbaum and Mallet-Paret (1996)] J. Mallet-Paret and R. D. Nussbaum, Boundary layer phenomena for differential-delay equations with state-dependent time lags II, J. Reine Angew. Math., 477 (1996), 129-197.

[Mallet-Paret et al. (1994)] J. Mallet-Paret, R. D. Nussbaum, P. Paraskevopoulos, Periodic solutions for functional-differential equations with multiple state-dependent time lags, Topol. Methods Nonlinear Anal. 3 (1994), no. 1, 101-162.

[Rezounenko (1997)] A. V. Rezounenko, On singular limit dynamics for a class of retarded nonlinear partial differential equations, Matematicheskaya fizika, analiz, geometriya, 4 (1/2), (1997), 193-211.

[Rezounenko (2003)] A.V. Rezounenko, Approximate inertial manifolds for retarded semilinear parabolic equations, Journal of Mathematical Analysis and Applications, 282 (2) (2003), 614-628.

[Rezounenko and Wu (2006)] A.V. Rezounenko and J. Wu, A non-local PDE model for population dynamics with state-selective delay: local theory and global attractors // Journal of Computational and Applied Mathematics (2006) Vol. 190, Issues 1-2, P.99-113.

[Rezounenko (2007)] A.V. Rezounenko, Partial differential equations with discrete and distributed state-dependent delays // Journal of Mathematical Analysis and Applications (2007) Vol. 326, Issue 2, P.1031-1045. (see also detailed preprint, March 22, 2005, http://arxiv.org/pdf/math.DS/0503470 ).

[So, Wu and Yang (2000)] J. W. -H. So, J. Wu and Y. Yang, Numerical steady state and Hopf bifurcation analysis on the diffusive Nicholson's blowflies equation. Appl. Math. Comput. 111 (2000), no. 1, 33-51.

[So, Wu and Zou (2001)] J. W. -H. So, J. Wu and X.Zou, A reaction diffusion model for a single species with age structure. I. Travelling wavefronts on unbounded domains, Proc. Royal .Soc. Lond. A (2001) 457, 1841-1853.

[So and Yang (1998)] J. W.- H. So and Y. Yang, Dirichlet problem for the diffusive Nicholson's blowflies equation, J. Differential Equations 150 (1998), no. 2, 317348. 
[Temam (1988)] R. Temam, Infinite Dimensional Dynamical Systems in Mechanics and Physics, Springer, Berlin-Heidelberg-New York, 1988.

[Travis and Webb (1974)] C. C. Travis and G. F. Webb, Existence and stability for partial functional differential equations, Transactions of AMS 200, (1974), 395418.

[Walther (2002)] H. -O. Walther, Stable periodic motion of a system with state dependent delay, Differential and Integral Equations 15 (2002), 923-944.

[Walther (2003)] H.-O. Walther, The solution manifold and $C^{1}$-smoothness for differential equations with state-dependent delay, J. Differential Equations 195 (2003), no. $1,46-65$.

[Walther (2007)] H.-O. Walther, On a model for soft landing with state-dependent delay, 19 (3), (2007), 593-622.

[Winston (1970)] E. Winston, Uniqueness of the zero solution for differential equations with state-dependence, J. Differential Equations 7 (1970), 395-405.

[Wu (1996)] J. Wu, Theory and Applications of Partial Functional Differential Equations, Springer-Verlag, New York, 1996.

[Yosida (1965)] K. Yosida, Functional analysis, Springer-Verlag, New York, 1965.

Kharkiv

February 4, 2008 